\title{
The orientation independence of the CdTe-HgTe valence band offset as determined by $x$-ray photoelectron spectroscopy
}

\author{
C R Becker, Y S Wu†, A Waag, M M Kraus and G Landwehr \\ Physikalisches Institut der Universität Würzburg, D-8700 Würzburg, Federal \\ Republic of Germany
}

\begin{abstract}
Absiract. We have investigated the valence band offset $\left(\Delta E_{\mathrm{v}}\right)$ of the CdTe-HgTe heterojunction for three orientations, (100), (110) and (111)B, using in situ $x$-ray photoelectron spectroscopy. The difference in energy between the $\mathrm{Cd} 4 \mathrm{~d}$ and $\mathrm{Hg}$ $5 d_{5 / 2}$ core levels, $\Delta E_{C L}$, and consequently $\Delta E_{V}$ was found to be independent of surface orientation and the surface structure immediately prior to growth of the uppermost layer. $\Delta E_{\mathrm{V}}$ was found to be $0.37 \pm 0.07 \mathrm{eV}$.
\end{abstract}

\section{Introduction}

The valence band offset $\left(\Delta E_{v}\right)$ of an abrupt CdTe-HgTe heterojunction is an important parameter for the fabrication of devices as well as having a large effect on the band structure of quantum wells and superlattices (see, for example, Meyer et al [1]). $\Delta E_{\mathrm{v}}$ for the CdTe-HgTe interface has recently been the subject of a certain amount of controversy. The common anion rule predicts a small valence band offset which was initially corroborated by a magneto-optical investigation by Guldner et al [2], i.e. their measurements were consistent with a valence band offset of $40 \mathrm{meV}$, However, a much larger value of $350 \mathrm{meV}$ was reported later by two separate $x$-ray photoelectron spectroscopy (XPS) investigations $[3,4]$. The valence band offset was also found to be independent of growth sequence and the thickness of the uppermost layer from 5 to $35 \AA$ [4]. In addition, XPS and ultraviolet photoemission spectroscopy (UPS) measurements by Sporken et al [5] have shown that the valence band offset is temperature independent and therefore the discrepancy between these two values can not be explained as the result of a temperature dependence. The ensuing controversy, which has been extensively reviewed by Meyer et al [1], appears to be resolved in favour of the larger value.

Self-consistent tight-binding (SCTB) calculations by Muñoz et al [6] have predicted a large dependence on orientation, $180 \mathrm{meV}$ between the (100) and (110) surfaces $\left(\Delta E_{\mathrm{v}}=0.46\right.$ and $0.28 \mathrm{eV}$, respectively). In contrast, † Permanent address: Institute of Physics, Chinese Academy of
Science, Beijing, People's Republic of China.
Van de Walle et al [7], using self-consistent local density functional methods, predict no surface dependence, i.e. $\Delta E_{\mathrm{V}}=0.27$ and $0.28 \mathrm{eV}$, respectively. In fact a more general study by the latter authors suggests that this independence is a characteristic of a number of important interfaces, e.g. CdTe-HgTe, AlAs-GaAs and Si-Ge. Indeed this has been shown to be the case for the GaAs-AlAs heterojunction [8]. More recent calculations by Muñoz et al [9] resulted in a much smaller dependence on orientation, $90 \mathrm{meV}$ between the $(100)$ and (110) surfaces $\left(\Delta E_{\mathrm{V}}=0.46\right.$ and $0.37 \mathrm{eV}$, respectively). In order to determine which is correct for the CdTe-HgTe heterojunction we have investigated the effect of surface orientation as well as the effect of interface structure on the valence band offset.

\section{Experimental details}

Epitaxial growth was carried out in a four-chamber RIBER 2300, molecular beam epitaxial (MBE) system which has been modified to permit the growth of $\mathrm{Hg}$ based materials. The vacuum in the growth chamber is better than $6 \times 10^{-10}$ Torr when no $\mathrm{Hg}$ has recently been admitted. Three MBE cells were employed, two of which were commercial cells and which contained highpurity $\mathrm{CdTe}$ and $\mathrm{Te}$. The third cell, designed by us, is a stainless steel cell for $\mathrm{Hg}$ which can be refilled without breaking the vacuum. The flux of the latter cell is stable to within \pm 1.5 and $\pm 3 \%$ over a period of 2 and $30 \mathrm{~h}$, respectively. The growth chamber is connected with the XPS chamber $\left(3 \times 10^{-10}\right.$ Torr $)$ with a transfer system whose vacuum was better than $1 \times 10^{-9}$ Torr. 
The CdTe-HgTe heterojunctions were grown on (110) CdTe and on (100)- and (111)B-oriented CdTe and $\mathrm{CdZnTe}$ substrates which had been degreased, chemomechanically polished for several minutes, etched in a weak solution of bromine in methanol and rinsed in methanol. Immediately prior to loading the substrates into the MBE system, they were rinsed in de-ionized water, briefly dipped in hydrochloric acid and then rinsed in deionized water so as to remove all of the original oxide and carbon from the substrate surface. We have found that, as a result of this previous step, the newly formed oxide, is much more easily evaporated from the surface. This is accomplished by heating the substrates at temperatures up to about $350^{\circ} \mathrm{C}$ while being monitored by reflection high-energy electron diffraction (RHEED) as described elsewhere [10]. Throughout this paper we consistently use the convention of referring to the direction of the incident electrons when referring to reconstruction in a particular azimuth.

Approximately 0.1 and $2 \mu \mathrm{m}$ of CdTe were grown on $\mathrm{CdTe}$ and $\mathrm{CdZnTe}$ substrates, respectively. This growth was initiated at 300 and $340{ }^{\circ} \mathrm{C}$, respectively and continued while lowering the temperature to $230^{\circ} \mathrm{C}$ where the growth was completed. Then a thin layer, $6-40 \AA$, of $\mathrm{HgTe}$ was grown at $180^{\circ} \mathrm{C}$. For the (100) and (111)B orientations, this thin layer of $\mathrm{HgTe}$ was on a Testabilized surface as well as a surface displaying attributes of both Te and $\mathrm{Cd}$ stabilization. The former surface structure was established by exposing the CdTe film to a Te flux of $3 \times 10^{-7}$ Torr at $210^{\circ} \mathrm{C}$. The latter surface structure was established by evaporating $\mathrm{Te}$ from the surface at about $340^{\circ} \mathrm{C}$ for several minutes while maintaining a smooth surface as evidenced by the presence of uniform streaks and the absence of spots in the RHEED pattern. In the (100) case this mixture of $\mathrm{Te}$ and $\mathrm{Cd}$ stabilization of this surface is characterized by half-order reconstruction in the [011] and [010] azimuths. The (111)B surface is more complicated. Here the $\mathrm{Te}(1 \times 1)$ stabilized surface undergoes a transition to $(2 \sqrt{3} \times$ $2 \sqrt{3}) R 30^{\circ}$ reconstruction upon evaporation of Te from

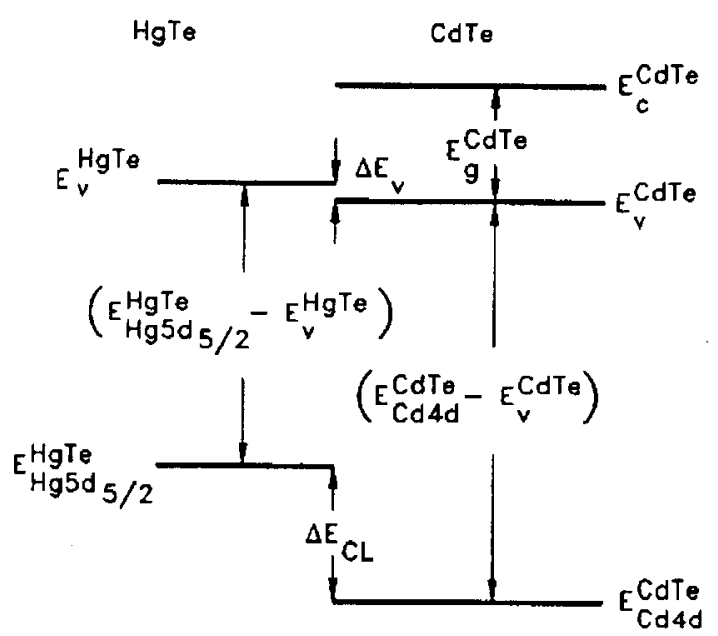

Figure 1. A schematic diagram of the relevant energy levels of an abrupt CdTe-HgTe heterojunction. the surface. The as-grown surface structure in the (110) orientation could not be changed either with excess Te flux or with an increase in temperature up to $340^{\circ} \mathrm{C}$, and therefore $\mathrm{HgTe}$ was grown only on the as-grown $\mathrm{CdTe}$ surface.

The resulting heterojunctions were transferred via an ultra-high-vacuum transfer system and investigated by XPS under nearly in situ conditions. XPS experiments were preformed with a RIBER MAC 2 electron spectrometer using an $\mathrm{Mg} \mathrm{K} \alpha \mathrm{x}$-ray source $(1253.6 \mathrm{eV})$ with an acceleration voltage of $10 \mathrm{kV}$, a current of $10 \mathrm{~mA}$ and without a monochromator. The energy scans were repeated for at least $12 \mathrm{~h}$ in order to achieve an acceptable signal-tonoise ratio.

\section{Results and discussion}

The valence band offset $\Delta E_{\mathbf{V}}$ is schematically shown in figure 1 and is given by

$\Delta E_{\mathrm{V}}=\left(E_{\mathrm{Gg}}^{\mathrm{Hg} \mathrm{Hec}^{2} / 2}-E_{\mathrm{V}}^{\mathrm{HgTe}}\right)-\left(E_{\mathrm{Cd} 4 \mathrm{~d}}^{\mathrm{Cde}}-E_{\mathrm{V}}^{\mathrm{CdTe}}\right)+\Delta E_{\mathrm{CL}} \cdot(1)$

Therefore, in order to determine $\Delta E_{\mathrm{v}}$, we have to measure these three binding energy differences for $\mathrm{HgTe}$, $\mathrm{CdTe}$ and the CdTe-HgTe heterojunction. Where $E_{\mathrm{Hg}}^{\mathrm{HgT} \mathrm{He}_{5 / 2}}$ and $E_{\mathrm{Cd} 4 \mathrm{~d}}^{\mathrm{CdTe}}$ are binding energies of the $\mathrm{Hg} 5 \mathrm{~d}_{5 / 2}$ and $\mathrm{Cd} 4 \mathrm{~d}$ core levels in $\mathrm{HgTe}$ and $\mathrm{CdTe}$, respectively. $E_{\mathrm{V}}^{\mathrm{Hg} T e}$ and $E_{\mathrm{V}}^{\mathrm{CdTe}}$ are the energies of the valence band maxima in $\mathrm{HgTe}$ and $\mathrm{CdTe}$, respectively, and $\Delta E_{\mathrm{CL}}$ is the binding energy difference between the $\mathrm{Hg} 5 \mathrm{~d}_{5 / 2}$ and $\mathrm{Cd}$ $4 \mathrm{~d}$ core levels in the $\mathrm{HgTe}-\mathrm{CdTe}$ heterojunction. This procedure results in a value of $0.37 \pm 0.07 \mathrm{eV}$ for $\Delta E_{\mathrm{V}}$ for the $(100)$ orientation. The large uncertainty is due primarily to the difficulty in determining the position of the valence band maximum (see figure 2). As can be seen by comparing the XPS spectra for (100) and (110) CdTe in figure 2 , the energy difference between the $\mathrm{Cd} 4 \mathrm{~d}$ core level and valence band maximum in CdTe is independent of these two surface orientations. The same is true for

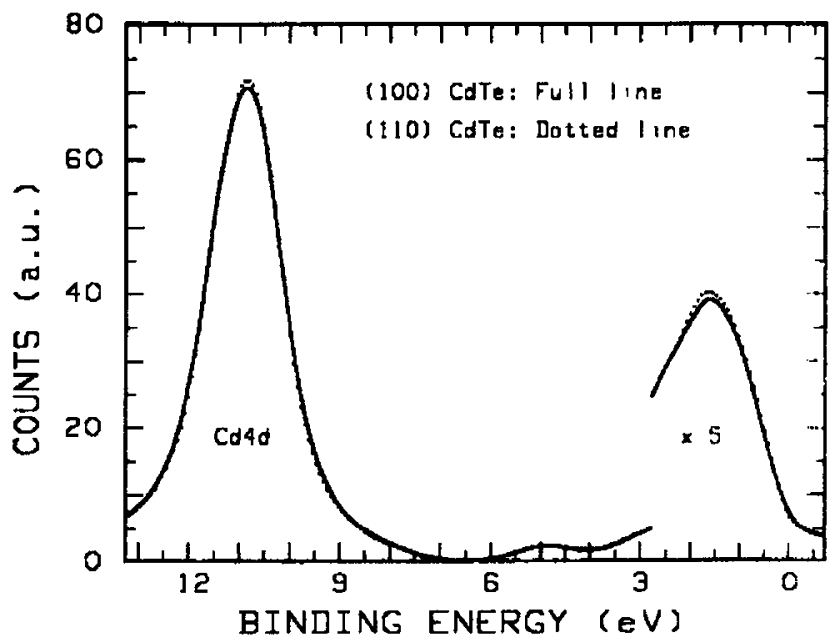

Figure 2. XPS spectra of (100) and (110) CdTe, showing the Cd $4 \mathrm{~d}$ core level peak and the CdTe valence band maximum. 


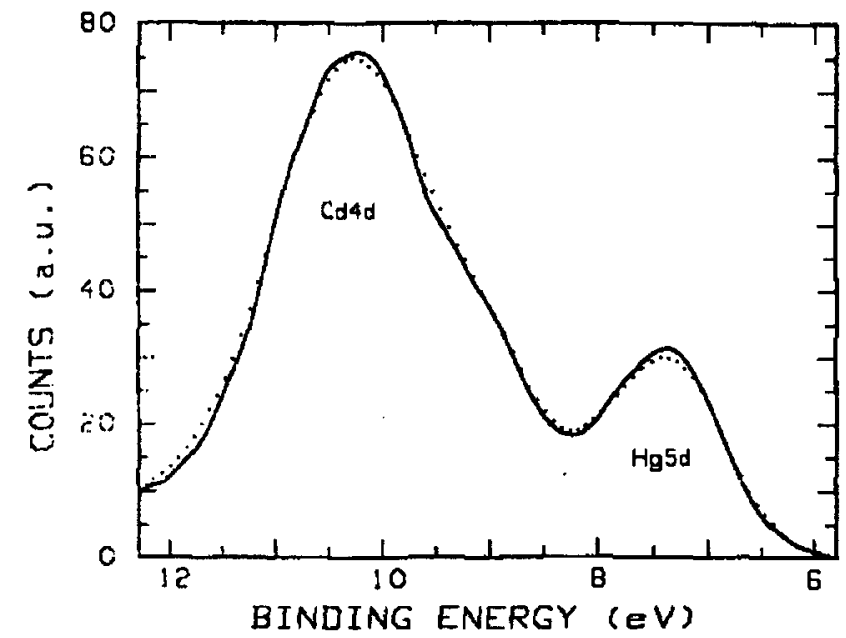

Figure 3. An XPS spectrum of a (110) CdTe-HgTe heterojunction (full curve) and a least-squares fit of the XPS spectra of a (110) CdTe and (110) $\mathrm{HgTe}$ film (dotted curve) in the vicinity of the $\mathrm{Cd} 4 \mathrm{~d}$ and $\mathrm{Hg} 5 \mathrm{~d}$ core levels.

(111)B. This demonstrates that this energy difference is a bulk property and is independent of orientation as is usually assumed. Consequently all orientation or interface effects on $\Delta E_{\mathrm{v}}$ are contained in $\Delta E_{\mathrm{CL} \text {. }}$ which can be determined with greater precision than the position of the valence band.

An XPS spectrum for a (110) CdTe-Hg Te heterojunction is shown in figure 3 and the corresponding spectra for $\mathrm{CdTe}$ and $\mathrm{HgTe}$ epitaxial films in the region of the $\mathrm{Cd}$ $4 d$ and $\mathrm{Hg} 5 \mathrm{~d}$ core levels can be seen in figure $4 . \Delta E_{\mathrm{CL}}$ cannot be determined directly for the heterojunction due to the overlapping of the $\mathrm{Cd} 4 \mathrm{~d}, \mathrm{Hg} 5 \mathrm{~d}_{3 / 2}$ and $\mathrm{Hg} 5 \mathrm{~d}_{5 / 2}$ core levels. First the $\mathrm{HgTe}$ and $\mathrm{CdTe}$ spectra were combined and fitted to the heterojunction spectrum by means of a least-square procedure. This least-square fit as well as the heterojunction spectrum are plotted in figure 3 . In order to more accurately determine the position of the peak

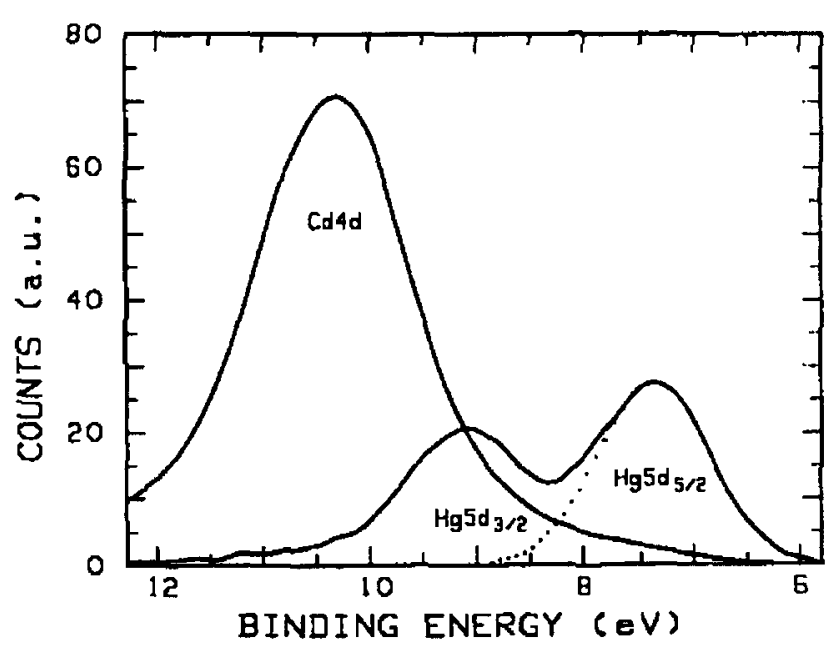

Figure 4. xps spectra of (110) CdTe and (110) HgTe films in the vicinity of the $\mathrm{Cd} 4 \mathrm{~d}$ and $\mathrm{Hg} 5 \mathrm{~d}$ core levels.
Table 1. The energy difference between the $\mathrm{Cd} 4 \mathrm{~d}$ and $\mathrm{Hg} 5 \mathrm{~d}_{5 / 2}$ core levels for the (100), (110) and (111)B orientations.

\begin{tabular}{ll}
\hline Orientation & $\Delta E_{\mathrm{CL}}(\mathrm{eV})$ \\
\hline$(100)$ & $2.96 \pm 0.03$ \\
$(110)$ & $3.01 \pm 0.03$ \\
$(111) \mathrm{B}$ & $2.98 \pm 0.03$ \\
\hline
\end{tabular}

due to the $\mathrm{Hg} 5 \mathrm{~d}_{5 / 2}$ core level it was resolved from the HgTe spectrum as depicted by the dotted curve in figure 4. This was accomplished by assuming that this peak is symmetrical, i.e. that the high-energy flank is a mirror image of the low-energy flank. This should be a good assumption because only minimal inelastic scattering. is expected under the low-energy flank. $\Delta E_{\mathrm{CL}}$ was then determined by measuring the energy difference between the peak centres at half maximum of the $\mathrm{Cd} 4 \mathrm{~d}$ and $\mathrm{Hg}$ $5 d_{5 / 2}$ core levels.

The results for the (100), (110) and (111)B orientations are tabulated in table 1. Two different interface structures were investigated for the (100) and (111)B orientations. In the first case $\mathrm{HgTe}$ was grown on $(100)-(2 \times 1)$ and $(111) B-(1 \times 1)$ Te-stabilized CdTe surfaces respectively, whereas in the second case HgTe was grown on a mixed surface with half-order reconstruction in both the [011] and [010] azimuths for the (100) orientation and $(2 \sqrt{3} \times 2 \sqrt{3}) R 30^{\circ}$ reconstruction for (111)B. In each case the two corresponding values are the same within the experimental uncertainities given in table 1 . As can be seen in table $1, \Delta E_{\mathrm{CL}}$ and therefore $\Delta E_{\mathrm{V}}$ for the CdTe-HgTe heterojunction is, within experimental uncertainty $( \pm 0.03 \mathrm{eV})$, independent of orientation and interface structure.

\section{Conclusions}

In conclusion, by means of in situ XPS experiments we have shown that $\Delta E_{C L}$ (and therefore that $\Delta E_{v}$ ) for the $\mathrm{CdTe}-\mathrm{HgTe}$ heterojunction is independent of the surface orientation and the surface reconstruction immediately prior to the growth of $\mathrm{HgTe}$, whether Te-stabilized or a mixture of $\mathrm{Cd}$ and $\mathrm{Te}$ stabilization. These results agree with the self-consistent local density predictions of Van de Walle et al [7] but not with the SCTB calculations of Muñoz et al $[6,9]$. Furthermore, $\Delta E_{v}$ has been determined to be $0.37 \pm 0.07 \mathrm{eV}$, in good agreement with the literature [3-5].

\section{Acknowledgments}

This project was supported by the Bundesministerium für Forschung und Technlogie and the Deutsche Forschungsgemeinschaft. 


\section{References}

[1] Meyer J R, Hoffman C A and Bartoli F J 1990 Semicond. Sci. Technol. 5 S90

[2] Guldner Y, Bastard G, Vieren J P, Voos M, Faurie J P and Million A 1983 Phys. Rev. Lett. 51907

[3] Kowalczyk S P, Cheung J T, Kraut E A and Grant R W 1986 Phys. Rev. Lett. 561605

[4] Faurie J P, Hsu C and Duc T M 1987 J. Vac. Sci. Technol. A 53074

[5] Sporken R, Sivananthan S, Faurie J P, Ehlers D H, Fraxedas J, Ley L, Pireaux J J and Caudano R 1989

$$
\text { J. Vac. Sci. Technol. A } 7427
$$

[6] Muñoz A, Sanchez-Dehesa J and Flores F 1987 Phys. Rev. B 356468

[7] Van de Walle C G and Martin R M 1988 Phys Rev. B 374801

[8] Hirakawa K, Hashimoto Y and Ikoma T $1990 \mathrm{Appl}$. Phys. Lett. 572555

[9] Muñoz A, Sanchez-Dehesa J and Flores F 1988 Phys. Rev. B 374803

[10] Wu Y S, Becker C R, Waag A, Bicknell-Tassius R N and Landwehr G 1991 J. Appl. Phys. 69268 